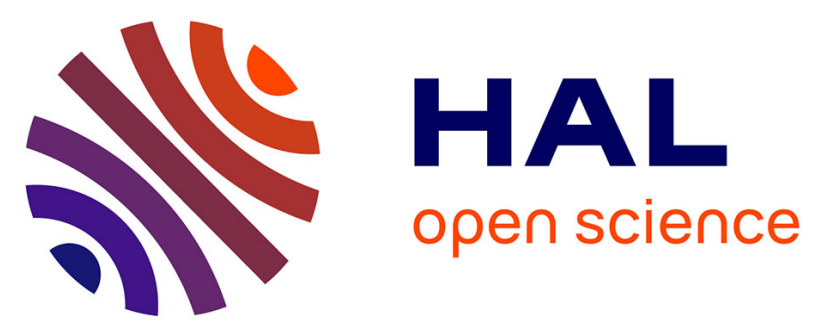

\title{
La perception gustative des populations autochtone de Yakoutie Arctique (District de Basse-Kolyma) en fonction du régime alimentaire, comparée à celle de populations d'Europe, d'Afrique et du Goroenland
} Christian Malet, Boris Chichlo, Joëlle Robert-Lamblin, Sandra Iaconelli, Patrick Pasquet, Claude Marcel Hladik

\section{To cite this version:}

Christian Malet,, Boris Chichlo, Joëlle Robert-Lamblin, Sandra Iaconelli, Patrick Pasquet, et al.. La perception gustative des populations autochtone de Yakoutie Arctique (District de Basse-Kolyma) en fonction du régime alimentaire, comparée à celle de populations d'Europe, d'Afrique et du Goroenland. Bulletins et Mémoires de la Société d'anthropologie de Paris, 1999, 11, pp.405-416. hal-00556266

\author{
HAL Id: hal-00556266 \\ https://hal.science/hal-00556266
}

Submitted on 12 Feb 2013

HAL is a multi-disciplinary open access archive for the deposit and dissemination of scientific research documents, whether they are published or not. The documents may come from teaching and research institutions in France or abroad, or from public or private research centers.
L'archive ouverte pluridisciplinaire HAL, est destinée au dépôt et à la diffusion de documents scientifiques de niveau recherche, publiés ou non, émanant des établissements d'enseignement et de recherche français ou étrangers, des laboratoires publics ou privés. 


\section{NOTES}

\section{LA PERCEPTION GUSTATIVE DES POPULATIONS AUTOCHTONES DE YAKOUTIE ARCTIQUE (DISTRICT DE BASSE-KOLYMA) EN FONCTION DU RÉGIME ALIMENTAIRE, COMPARÉE À CELLE DE POPULATIONS D'EUROPE, D'AFRIQUE ET DU GROENLAND}

Christian Malet ${ }^{1}$, Boris Chichlo ${ }^{1}$, Joëlle Robert-Lamblin ${ }^{1}$, Sandra Iaconelli², Patrick Pasquet $^{1,2}$, Claude Marcel Hladik ${ }^{2}$

Résumé.- Des tests en simple aveugle ont permis de mettre en évidence les seuils de reconnaissance gustative d'une série de produits purifiés, incluant le tannin de chêne et l'acide tannique, sur un échantillon de population $(n=109)$ de Yakoutie arctique, comprenant des Yakoutes $(\mathrm{n}=47)$, des personnes appartenant aux minorités autochtones du Grand Nord $(\mathrm{n}=38$, soit: 20 Evènes, 14 Youkaguirs, 3 Tchouktches et 1 Nénétse), ainsi que 24 personnes d'autres origines (Russes, Ukrainiens, etc.). Le même type de test ayant été effectué dans plusieurs pays d'Europe ainsi qu'en Afrique, nous avons mis en évidence des différences régionales significatives, portant en particulier sur la perception des sucres, discutées en fonction du milieu et des adaptations au régime alimentaire. Dans ce contexte, la perception du chlorure de sodium par les populations de Yakoutie n'est que partiellement comparable à celle des Inuit du Groenland, car la très grande acuité de perception de ces derniers n'a pas été retrouvée, ce qui impliquerait une adaptation au milieu continental plutôt qu'aux régions côtières arctiques.

Mots-clés : seuils de perception gustative, tannins, sucres, chlorure de sodium, Yakoutie, sociétés arctiques.

GUSTATORY PERCEPTION OF ABORIGENAL POPULATIONS OF THE SAKHA REPUBLIC (YAKUTIA), LOWER KOLYMA DISTRICT, IN RELATION TO DIET, AS COMPARED TO POPULATION SAMPLES FROM EUROPE, AFRICA AND GREENLAND

Summary. - Thresholds of taste recognition have been determined among a Yakutian population sample $(n=109)$, including Yakuts $(n=47)$, some people belonging to aborigenal ethnic minoritie of the North ( $\mathrm{n}=38$, including 20 Evens, 14 Yukagirs, 3 Chukchi and 1 Nenets), and 24 persons of

1. E.P. 1781 du CNRS (Dynamique de l'Évolution Humaine), 44 Rue de l'Amiral Mouchez, 75014 Paris e-mail christian.malet@libertysurf.fr

2. E.P. 2017 du CNRS (Éco-Anthropologie: Environnements, Individus et Sociétés), Laboratoire d’Écologie Générale du MNHN. 4 avenue du Petit Château, 91800 Brunoy; e-mail : hladik@ @cr.jussieu.fr. 
other origin (Russians, Ukrainians, etc.), using a blind protocol with solutions of pure (or purified) substances, including oak tanin and tannic acid. Significant differences for sugars perception have been found when comparing the results with those obtained (with a similar protocol) in various European countries and in Africa. These differences are discussed in relation to the environmental conditions and dietary adaptations. In this context, the reactions to sodium chloride, although partly analogous in the Yakutian population sample and among the Inuit of Greenland, do not present the same degree of acuity. This difference could result of an environmental continental adaptive trend, whereas the Inuit have adapted to the arctic coast environmental conditions.

Keywords : taste perception thresholds, tanins, sugars, sodium chloride, Sakha Republic (Yakutia), Arctic societies.

\section{INTRODUCTION}

Dans le cadre d'un programme de recherche portant sur les changements anthropobiologiques et socioculturels des sociétés arctiques, nous avons entrepris une série de tests portant sur la discrimination gustative de solutions de produits purs (ou purifiés, en ce qui concerne les tannins), en parallèle avec l'étude des habitudes alimentaires (RobertLamblin, 1998) et une enquête généalogique et démographique d'un échantillon de population de Yakoutie du district de Basse-Kolyma ${ }^{(1)}$.

Le régime alimentaire des populations autochtones de la Basse-Kolyma a toujours été constitué essentiellement de protéines animales (viande et poisson) et l'apport végétal y est extrêmement réduit. L'arrivée des cosaques russes au XVIIe siècle (Chichlo, 1997, 1999a) n'a pas modifié ces pratiques alimentaires. Au contraire, ce sont les envahisseurs qui ont fini par les adopter. Leurs descendants, formant un groupe ethnique distinct, ont privilégié la pêche et sont devenus de véritables piscivores, certains répétant les paroles de leurs pères: «Ryba èto nach khleb» («le poisson est notre pain»). Parmi les nouveaux produits auxquels ont pris goût les autochtones depuis les premiers contacts avec les Russes, au point de ne pouvoir s'en passer, figurent: le thé, le tabac et l'alcool (vodka); mais ceux-ci ne parvenaient dans la région que de façon irrégulière.

Les pratiques alimentaires de la population autochtone n'ont subi de transformation radicale qu'à partir des années 1950, avec l'afflux des migrants venus de toutes les parties de l'ex-URSS ; ceux-ci, une fois devenus majoritaires, ont renversé la situation démographique du district de Basse-Kolyma et provoqué une distorsion entre les cultures environnementalistes et un type de civilisation coercitif (Chichlo, 1999b). L'approvisionnement régulier par le port de Tcherski en produits alimentaires jusque là peu accessibles,

1. Cette mission, financée par l'Institut Français de Recherche et de Technologie Polaires (I.F.R.T.P., Brest), a été effectuée avec la collaboration de l'Institut des Problèmes des Minorités du Nord de Yakoutsk (Pr. V. Robbek). Que ces deux institutions soient remerciées pour toute l'aide qu'elles nous ont apportée dans la réalisation de nos travaux. voire inconnus, l'accoutumance des enfants aux menus des cantines scolaires dans les internats obligatoires, la progression croissante des mariages mixtes entre les femmes autochtones et les migrants — tous ces facteurs sont à l'origine des changements du comportement alimentaire. Toutefois, les produits venus de l'extérieur (en particulier céréales et conserves, fruits et légumes frais ou secs) se sont raréfiés dans le courant des années 1990, avec l'effondrement du système centralisé d'approvisionnement et la hausse du prix du transport.

Tous les nomades éleveurs de rennes de cette région, quelle que soit leur appartenance ethnique, se révèlent de très grands consommateurs de viande. Cette viande, provenant des rennes sauvages qu'ils chassent ou des rennes domestiques qu'ils élèvent, est souvent consommée bouillie. L'observation de la vie quotidienne dans un campement de renniculteurs (tchouktches, évènes et youkaguirs) nous a permis d'estimer la quantité de viande consommée journellement par les hommes adultes lorsqu'ils rentrent au campement ou lors de la garde du troupeau de renne. La quantité de viande ingérée quotidiennement, en plusieurs repas chauds ou froids, atteint entre $800 \mathrm{~g}$ et $1 \mathrm{~kg}$ par personne. On peut observer que ce régime alimentaire, très marqué par les aliments carnés, correspond à ce que l'on trouve par ailleurs chez les chasseurs de mammifères marins du Groenland oriental et chez les Yuit de Sibérie qui sont restés tournés vers les activités de chasse (Hubert et al., 1984 ; Robert-Lamblin, 1986; Krupnik, 1993; Robbe, 1994). En revanche, dans les familles dont l'activité principale est la pêche en rivière ou dans les lacs et la trappe aux animaux à fourrure en hiver, c'est le poisson (une quinzaine d'espèces) qui constitue l'essentiel de l'alimentation. Dans ce groupe, la viande de renne est complémentaire; elle fait l'objet d'échanges avec les éleveurs de renne.

Avant les contacts avec les Russes, les seuls aliments à goût sucré existant dans le milieu naturel étaient des baies (une dizaine d'espèces; Malet, 1995). Le sucre du commerce, fort apprécié et qui fait désormais partie du régime alimentaire quotidien, est consommé en grande quantité, notamment avec le thé, très fréquemment bu au cours de la journée.

Replacées dans ce contexte culturel, les données sur la perception gustative concernant une population du nord-est de la Yakoutie arctique (district de Basse-Kolyma) dans trois villages distants de plusieurs centaines de kilomètres les uns des autres permettent une approche anthropobiologique.

À titre comparatif, les données obtenues par deux d'entre nous (S.I. et P.P.), à l'aide du même protocole, dans divers pays de l'Union Européenne, au cours d'un projet de faisabilité( ${ }^{(2)}$ seront présentées. Ces données sur la perception gustative proviennent de petits échantillons de population, d'Italie, d'Espagne, de France, de Belgique et du Royaume-Uni mais concernent un total de 219 personnes (Iaconelli et al., 1998; Iaconelli, en prép.). Les résultats des mêmes types tests, réalisés au Cameroun (P.P.), sur un plus

2. Projet de l'U. E. n $97 / C A N / 45916$ « Consommer Méditerranéen : une attitude préventive au cancer », sou la responsabilité de Martine Padilla et Mariette Gerber. 
petit échantillon de population, seront également utilisés. Nous reprendrons par ailleurs les résultats publiés sur les Inuit de la côte est du Groenland (Hladik et al., 1986) pour comparer, en particulier, l'acuité de la perception du chlorure de sodium dans deux populations de l'Arctique, et proposer des hypothèses au sujet des similitudes et des différences observées.

\section{MATÉRIEL ET MÉTHODES}

Le principe de la détermination du seuil de reconnaissance repose sur la présentation, dans un ordre aléatoire, de produits simples (sucres, sel, acide citrique, quinine, tannin de chêne, etc.) en solution dans de l'eau utilisée localement pour la boisson. Pour chaque produit, une série de solutions va dans l'ordre croissant des concentrations doublées pas à pas : fructose (11 dilutions de 1 à $1000 \mathrm{mM} / \mathrm{l})$, saccharose ( 8 dilutions de 1,5 à $200 \mathrm{mM} /$ 1), chlorhydrate de quinine ( 11 dilutions, de 0,4 à $400 \mu \mathrm{M} / \mathrm{l})$, chlorure de sodium (10 dilutions de 0,5 à $250 \mathrm{mM} / \mathrm{l}$ ), acide citrique ( 8 dilutions de 0,2 à $25 \mathrm{mM} / \mathrm{l})$, acide tannique (11 dilutions de 4 à $8000 \mu \mathrm{M} / \mathrm{l})$, tannin de chêne (10 dilutions de 0,03 à $8 \mathrm{~g} / \mathrm{l})$. Seules deux dilutions de n6-propyl-thiouracile (PROP) — respectivement à 0,1 et $0,2 \mathrm{mM} / 1$ — ont été utilisées pour estimer la fréquence des «goûteurs» et des «non goûteurs». Toutes les solutions, utilisées à température ambiante, étaient renouvelées régulièrement.

Partant toujours des solutions les plus diluées, pour mettre en bouche, avant de recracher, un petite cuillère (volume : $2 \mathrm{à} 3 \mathrm{~cm}^{3}$ ) de chaque produit, les premières stimulations correspondent au goût de l'eau pure. L'ordre de présentation des solutions est, en fait, pseudoaléatoire (afin d'avoir une procédure standard simple pour l'expérimentateur qui suit une liste pré-établie). Cela correspond à un test en simple aveugle pour la personne qui goûte et doit donner une réponse sans savoir de quelle substance il s'agit, parmi différents goûts possibles. Après avoir recraché la solution, le sujet décrit le goût perçu (eau pure, sucré, salé, acide, amer, goût astringent ou autre). Entre chaque présentation, le sujet se rince la bouche avec de l'eau à température ambiante. Le seuil de reconnaissance correspond à la concentration de la première des solutions dont le goût est reconnu, après un test complémentaire avec les solutions de concentration immédiatement supérieure et inférieure.

L'échantillon de population testé (total de 109 personnes, dont 91 femmes) comprend des Yakoutes $(\mathrm{n}=47)$, des personnes appartenant aux minorités autochtones du Grand Nord $(n=38$, soit: 20 Evènes, 14 Youkaguirs, 3 Tchouktches et 1 Nénétse), ainsi que 24 personnes d'autres origines (Russes, Ukrainiens, etc.). En Yakoutie, l'une des difficultés majeures est la polyethnicité et une polyglossie élevées : douze ethnies ont été recensées, qui théoriquement s'expriment en autant de langues différentes (Malet, 1990). Le russe et le yakoute ont servi de langues véhiculaires mais la compréhension du test et la détermination exacte des saveurs perçues ont nécessité des explications plus ou moins longues. Il convient néanmoins de préciser, que tous les tests ayant été conduits par la même personne (C.M.), il n'existe pas de divergence dans l'interprétation des réponses obtenues.
Les analyses des résultats portent généralement sur les 109 sujets testés en Yakoutie; mais un nombre plus faible de sujets a été retenu pour certaines substances (en particulier pour les tannins), lorsque certaines réponses étaient trop ambigües pour être comptabilisées. L'analyse statistique des résultats a été faite par la méthode des probits (Finney 1971) qui permet d'estimer les moyennes théoriques des seuils de reconnaissances dans les différentes populations, à l'aide de la procédure PROBIT du logiciel SAS (SAS Institute, 1994). Les différences entre groupes sont discutées en fonction d'une analyse unimodale de la variance en utilisant le logiciel LeBayesien (Lecoutre et Poitevineau, 1996) qui permet, en outre, l'utilisation de procédures bayesiennes afin d'obtenir une estimation probabiliste des différences $(\delta)$ entre les populations, en fonction des échantillons testés. À cet égard, nous avons repris les conventions établies par Cohen (1969), et considéré les valeurs limites de l'effet calibré $(\delta / \sigma)$ de 0,2 et de 0,8 écart-type comme définissant respectivement un effet faible et un effet important.

\section{RÉSULTATS}

Les résultats des tests de reconnaissance gustative de Yakoutie sont présentés dans le Tableau I, dans lequel nous avons également inclus, à titre comparatif, les résultats obtenus, avec le même type de test et les mêmes lots de produits purifiés, sur des populations européennes et sur un échantillon de population africaine (Yaoundé, Cameroun).

Les principales différences entre populations sont illustrées par la Figure 1, montrant les pourcentages cumulés des sujets des différentes populations percevant le goût des substances pour différentes concentrations. Ces différences portent sur la perception des sucres : pour le saccharose, les différences sont significatives $\left[\mathrm{F}_{(2,352)}=24,43 ; \mathrm{p}=0,000\right]$ et la procédure bayesienne montre un effet important lorsqu'on compare l'échantillon de Yakoutie à celui du Cameroun $\left[\mathrm{p}_{(\delta / \sigma>0,8)}=0,86\right]$ que l'on retrouve, dans une moindre mesure, en comparant Yakoutie et Cameroun + Europe $\left[\mathrm{p}_{(0,69<\delta / \sigma<1,14)}=0,9\right]$; pour le fructose, les différences sont également significatives $\left[\mathrm{F}_{(2,346)}=27,79 ; \mathrm{p}=0,000\right]$ avec un effet calibré important en comparant l'échantillon de Yakoutie avec le Cameroun ou l'ensemble Cameroun + Europe [respectivement $\mathrm{p}_{(\delta / \sigma>0,8)}=0,99$ et $\left.\mathrm{p}_{(\delta / \sigma>0,8)}=0,94\right]$. Les différences entre les seuils moyens de reconnaissance des sucres, plus élevés en Yakoutie (20 et 37 mM/l, respectivement pour le saccharose et le fructose), correspondant à une moindre sensibilité, doivent donc être retenues. En revanche, en ce qui concerne les goût amer du chlorhydrate de quinine, aucune différence n'apparaît entre les échantillons de population testés $\left[\mathrm{F}_{(2,305)}=0,041 ;\right.$ non significatif $]$.

Pour la perception du chlorure de sodium, les différences entre populations sont significatives $\left[\mathrm{F}_{(2,347)}=10,03 ; \mathrm{p}=0,000\right]$ avec un effet de moyenne ampleur lorsqu' on compare la Yakoutie à l'Europe $\left[\mathrm{p}_{(0,2<\delta / \sigma<0,8)}=0,94\right]$, mais qui n'est pas importante $\left[\mathrm{p}_{(\delta / \sigma<0,8)}=0,99\right]$ et 


\begin{tabular}{|c|c|c|c|c|c|}
\hline & $\begin{array}{r}\text { Effectif } \\
n=\end{array}$ & $\begin{array}{r}\text { Seuil } \\
\text { moyen }\end{array}$ & $\begin{array}{r}\text { Seuil } \\
(\log )\end{array}$ & $\begin{array}{r}\text { Écart- } \\
\text { type }\end{array}$ & $\begin{array}{c}\text { Intervalle } \\
\text { fiduciaire à } 95 \%\end{array}$ \\
\hline \multicolumn{6}{|c|}{ Saccharose (mM/l) } \\
\hline Yakoutie & 109 & 20,00 & 1,30 & 0,40 & $1,19-1,41$ \\
\hline Yaoundé & 30 & 8,00 & 0,90 & 0,34 & $0,82-0,99$ \\
\hline Europe & 216 & 10,98 & 1,04 & 0,34 & $1,00-1,07$ \\
\hline \multicolumn{6}{|c|}{ Fructose (mM/l) } \\
\hline Yakoutie & 108 & 37,14 & 1,57 & 0,41 & $1,52-1,62$ \\
\hline Yaoundé & 30 & 10,27 & 1,01 & 0,37 & $0,92-1,10$ \\
\hline Europe & 211 & 20,11 & 1,30 & 0,41 & $1,27-1,34$ \\
\hline \multicolumn{6}{|c|}{ Chlorure de sodium (mM/l) } \\
\hline Yakoutie & 101 & 10,09 & 1,00 & 0,57 & $0,94-1,06$ \\
\hline Yaoundé & 30 & 6,89 & 0,84 & 0,47 & $0,73-0,93$ \\
\hline Europe & 219 & 15,77 & 1,19 & 0,45 & $1,16-1,23$ \\
\hline \multicolumn{6}{|c|}{ Quinine (mM/l) } \\
\hline Yakoutie & 93 & 16,26 & 1,21 & 0,45 & $1,11-1,31$ \\
\hline Yaoundé & 30 & 16,47 & 1,22 & 0,64 & $1,09-1,34$ \\
\hline Europe & 185 & 17,18 & 1,23 & 0,58 & $1,14-1,32$ \\
\hline \multicolumn{6}{|c|}{ Acide citrique $(\mathrm{mM} / \mathrm{l})$} \\
\hline Yakoutie & 93 & 0,83 & $-0,08$ & 0,49 & $-0,14--0,019$ \\
\hline Yaoundé & 30 & 1,31 & 0,12 & 0,51 & $0,005-0,22$ \\
\hline Europe & 212 & 1,94 & 0,25 & 0,35 & $0,25-0,32$ \\
\hline \multicolumn{6}{|c|}{ Acide tannique $(\mathrm{mM} / \mathrm{l})$} \\
\hline Yakoutie & 32 & 384,53 & 2,58 & 0,71 & $2,44-2,69$ \\
\hline Yaoundé & 30 & 350,94 & 2,54 & 0,70 & $2,34-2,76$ \\
\hline Europe & 191 & 200,77 & 2,30 & 0,65 & $2,25-2,35$ \\
\hline \multicolumn{6}{|c|}{ Tannin de chêne $(\mathrm{mg} / \mathrm{l})$} \\
\hline Yakoutie & 93 & 444,45 & 2,64 & 0,65 & $2,54-2,75$ \\
\hline Yaoundé & 30 & 607,60 & 2,78 & 0,56 & $2,67-2,89$ \\
\hline Europe & 212 & 446,27 & 2,71 & 0,56 & $2,57-2,73$ \\
\hline
\end{tabular}

Tableau I.- Seuils de reconnaissance moyens, estimés par analyse des probits, pour les divers composés, en Yakoutie, au Cameroun (Yaoundé) et en Europe.

non significative $(\mathrm{t}=1,4 ; \mathrm{p}=0,16)$ entre l'échantillon de Yakoutie et celui de Yaoundé; ce dernier (avec un seuil moyen à $6,89 \mathrm{mM}$ ), montre la plus grande sensibilité au goût salé parmi les trois populations testées.

En ce qui concerne les tannins, les seuils moyens de reconnaissance sont généralement élevés en Yakoutie (moindre sensibilité aux tannins) mais les différences, bien que

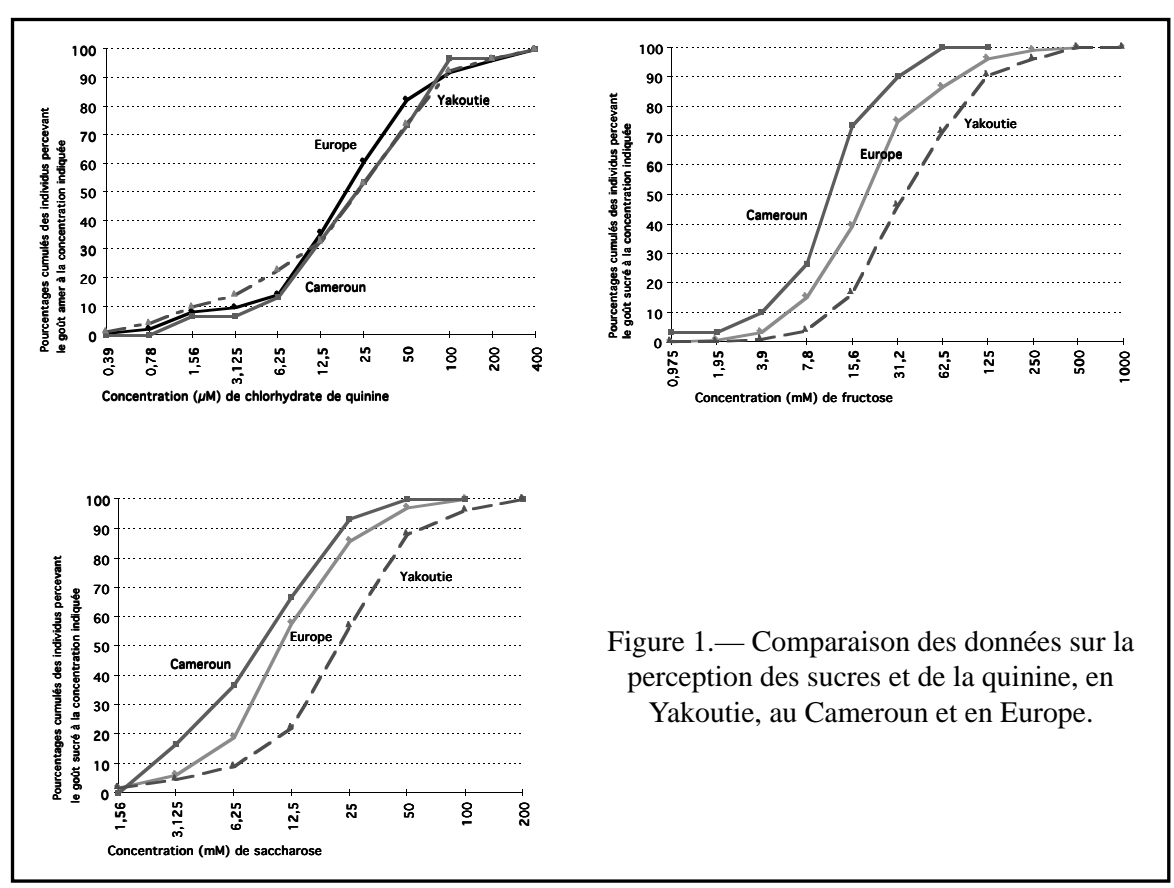

significatives entre populations pour l'acide tannique $\left[\mathrm{F}_{(2,250)}=3,64 ; \mathrm{p}=0,03\right]$ ne sont pas importantes, d'après l'analyse bayesienne, lorsqu' on compare l'échantillon de population de Yakoutie avec celui d'Europe $\left[\mathrm{p}_{(\delta / \sigma<0,8)}=0,97\right]$ ou avec celui du Cameroun $\left[\mathrm{p}_{(\delta / \sigma<0,8)}=0,99\right]$. Les différences sont plus faibles pour le tannin de chêne $\left[\mathrm{F}_{(2,332)}=0,45\right.$; non significatif $]$. En revanche, les différences entre populations sont significatives pour la perception de l'acide citrique $\left[\mathrm{F}_{(2,32)}=21,13 ; \mathrm{p}=0,000\right]$, et loin d'être négligeables, les Yakoutes étant plus sensibles que les populations d'Europe $\left[\mathrm{p}_{(\delta / \sigma<0,2)}=0,99\right]$.

La perception du PROP qui permet de séparer, dans une population, des classes de «goûteurs» et de «non goûteurs» est l'un des seuls marqueurs génétiques actuellement bien connu dans le domaine de la gustation (voir Hladik et Pasquet, 1999). L'utilisation de deux solutions dont les concentrations se répartissent autour de l'antimode généralement observé (Drewnowski et Rock, 1995) permet de comparer les fréquences dans les différentes populations. Ainsi nous avons observé au cours de ces tests:

— en Yakoutie, 31 «non goûteurs» sur 94 tests validés (soit $33 \%$ de «non goûteurs »)

— au Cameroun (Yaoundé), 19 «non goûteurs » sur 99 tests (soit 19,2\%)

— en Europe, 19 «non goûteurs» sur 120 tests (soit 15,8\%). 
De ce point de vue, les Yakoutes se différencient significativement de l'échantillon européen $(p=0,0035)$, et de l'échantillon africain $(p=0,003)$; toutefois les tests qui ont pu être effectués à ce jour en Europe concernent davantage les régions méditerranéennes que l'Europe du Nord.

\section{DISCUSSION}

Les résultats nous amènent à évaluer une influence possible des conditions de l'environnement (considéré dans sa globalité, environnement physique et environnement socioculturel) sur la perception gustative; car les tendances observées sont toujours fortement marquées par les apprentissages et il est difficile de séparer la base biologique - liée aux conditions environnementales - des influences de la culture dans le déterminisme des perceptions gustatives (Simmen et Hladik, 1993).

Une des caractéristique des plus nettes qui apparaît en Yakoutie est la faible perception des sucres (seuils élevés), avec une variabilité intra-population comparable à celle des échantillons testés en Afrique et en Europe. Cette faible sensibilité au goût des sucres solubles est vraisemblablement à considérer parmi les réponses aux changements récents du régime alimentaire, qui crée une habituation à des taux de sucre élevés, plutôt qu'à une caractéristique biologique - dont on ne peut ignorer toutefois l'éventualité, lorsqu'on sait que la sensibilité au fructose est partiellement indépendante de celle du saccharose (Hladik et al., 1986).

Les différences portant sur les produits goûtant dans l'amer, en particulier les proportions de «goûteurs » et de «non goûteurs » au PROP sont évidemment à considérer parmi les marqueurs génétiques des populations, pouvant évoluer en fonction des pressions d'un environnement où les habitudes alimentaires jouent autant que la composition des espèces disponibles (voir la revue de Hladik et Pasquet, mentionnée ci-dessus).

L'introduction des solutions de tannins parmi les produits testés constitue une approche originale qui va plus loin dans l'analyse des perceptions. Les différences régionales observées pourraient correspondre aux teneurs en tannins dans les aliments qui ont été utilisés localement au cours des siècles passés. Mais les tannins, de même que de nombreuses autres substances sapides ne sont pas toujours considérés comme élicitant l'un des «goûts de base» mais plutôt comme faisant appel au sens tactile de la langue (impression de sécheresse provoquée par la précipitation des protéines salivaires). Avec l'avance de la recherche fondamentale et des théories de la perception gustative, il apparaît, en fait, que chaque substance sapide, incluant les tannins, a une «signature » qui lui est propre, beaucoup plus complexe que la simple combinaison de quelques goûts de base (Hladik et Simmen, 1996).

Indépendamment de ces considérations, la façon de percevoir les tannins (en particulier le tannin de chêne, utilisé dans nos tests) et les préférences locales constituent un marqueur culturel, d'autant plus que l'écorce de chêne est connue pour son utilisation en tisane (Bonneval et Malet, 1993). Certains sujets yakoutes en ont reconnu le goût au cours des tests. On remarque que ce tannin de chêne est perçu (en moyenne) avec une même acuité par les Yakoutes et les populations européennes (la population de Yaoundé étant moins sensible), alors que pour l'acide tannique, il existe une différence significative entre les populations européennes (seuil à $200 \mathrm{mM} / \mathrm{l}$ ) et l'ensemble des Camerounais et des Yakoutes, beaucoup moins sensibles (seuil supérieur à $350 \mathrm{mM} / \mathrm{l}$ ).

Nous remarquons également que la sensibilité au chlorure de sodium n'est pas, chez les Yakoutes, aussi grande que chez les Inuit du Groenland dont le seuil extrêmement bas (seuil médian proche de $5 \mathrm{mM} / \mathrm{l}$ pour les femmes) a été mis en évidence par Hladik et al. (1986). Comme dans le cas précédent, ces différences doivent être appréhendées à la fois d'un point de vue anthropobiologique et socioculturel. L'héritabilité éventuelle d'une grande sensibilité au sel a été discutée en fonction d'une possible pression de sélection de l'environnement côtier arctique (Simmen et Hladik, 1993). Elle va de pair avec un système de représentation du sel comme substance nocive (Robbe et Hladik, 1988). Il n'était pas possible d'établir un fait similaire chez les Tchouktches, en raison de leur faible représentation dans l'échantillon testé (seulement 3 individus). De plus, les risques de maladies cardiovasculaires sont plus liés chez eux au tabagisme, à la surconsommation en sucre et à l'alcoolisme qu'à une consommation excessive de chlorure de sodium (Malet, 1993, 1998).

La littérature ethnographique souligne l'absence de sel dans l'alimentation des Tchouktches et le rejet du goût salé par ces derniers. Encore aujourd'hui, dans les plus vieilles générations, ceux-ci se distinguent par leur aversion au sel. Au cours de notre enquête, plusieurs femmes âgées ont insisté sur le fait qu'elles utilisaient extrêmement peu de sel dans la cuisson des aliments. Nos collègues russes ont remarqué le même phénomène chez les nomades de la Tchoukotka; «Les hommes et les femmes de la vieille génération considèrent toujours que la viande perd son goût si elle est quelque peu salée » (Afanas'eva et Simtchenko, 1993). En fait, cette aversion pour le goût salé concernait l'ensemble des populations nomades de Sibérie (c'est à dire les Tchouktches, les Evènes, les Youkaguirs, et les Yakoutes devenus éleveurs de rennes, donc des populations à adaptation exclusivement continentale), si l'on se réfère aux notes de Wrangel (1843) qui a exploré la région de Nijne Kolymski en 1821: "Les nomades de la Sibérie ont la plus grande répugnance pour le sel et n'en font jamais usage.» (t. 1:274) et «Si l'étranger demande du sel, on lui en sert, mais jamais autrement; car les gens du pays ont pour ce assaisonnement le dégoût le plus prononcé.» (ibid, p. 163). En revanche, les plus jeunes générations ont vu leur goût évoluer à cet égard, probablement en raison de l'alimentation qu'ils ont reçue au cours de leur jeunesse lors de séjours en internat.

En dehors de ces faits qui ont marqué considérablement le comportement alimentaire et qui ont nécessairement une répercussion sur la perception (ne serait-ce que par l'entraînement et/ou l'habituation), la moindre différenciation observée chez les populations de Yakoutie arctique pourrait en partie résulter de leur caractère continental, avec une pression de l'environnement - sur la perception du chlorure de sodium aux très faibles concentrations - moindre que pour les populations Inuit des côtes de l'Océan Arctique. 


\section{BIBLIOGRAPHIE}

Afanas'eva (G.M.), Simtchenko (Y.B.), 1993. Tradicionnaja pichtcha beregovykh i olennykh tchuktchej. Narody i kultury, Vol XXIV, Sibirskij ètnografitcheskij sbornik, Institut d'Ethnographie et d'Anthropologie, Moscou, 6:56-100.

Bonneval (L. de), MaLEt (C.), 1993. Les plantes utilisées dans le passé et aujourd'hui [au Kamtchatka et dans la Tchoukotka]. Questions sibériennes, Sibérie III, pp. 251-288.

Сніснго (B.), 1997. What are the particular of relationships between the indigenous people of Siberia and Russia. Occasional Papers on Changes in the Slavic-Eurasian World. Hokkaido University, Slavic Research Center, Sapporo, 50: 48-54.

Chichlo (B.), 1999A. Sibérie: mode de colonisation - mode de production. Sibérie II. Questions sibériennes: Histoire, littérature, cultures. Institut d'Etudes Slaves, Paris, pp. 95-118.

Снісньо (В.), 1999в. Le district de Nijnekolymski : population, renniculture, ethnicité. Sibérie II. Questions sibériennes: Histoire, littérature, cultures. Institut d'Etudes Slaves, Paris, pp. 475-502.

Conen (J.), 1969. Statistical Power Analysis for the Behavioral Sciences. Academic Press, New York.

DREwnowsKi (A.), Rock (C.L.), 1995. The influence of genetic taste markers on food acceptance. Am. J. Clin. Nutr., 62: 506-511.

FINNEY (D.J.), 1971. Probit Analysis. A statistical treatment of the sigmoid response curve. Cambridge University Press, London.

HLADIK (C.M.), PASQUet (P.). 1999. Évolution des comportements alimentaires : adaptations morphologiques et sensorielles. Bull et Mém. de la Soc. d'Anthrop. de Paris, n.s., 11 : 307-332.

HLadik (C.M.), Robbe (B.), Pagezy (H.), 1986. Sensibilité gustative différentielle des populations Pygmées et non Pygmées de forêt dense, de soudaniens et d'Eskimos, en rapport avec l'environnement biochimique. Comptes Rendus des Scéances de l'Académie des Sciences, Paris, série III, 303: 453-458.

HLAdiK (C.M.), Simmen (B.), 1996. Taste perception and feeding behavior in nonhuman primates and human populations. Evolutionary Anthropology 5 : 58-71.

Hubert (A.), Robert-Lamblin (J.), Hermann (M.), 1984. Environmental factors, dietary behaviors, and nasopharyngeal carcinoma: anthropological approach. In : R. Fortuine (Ed.), Circumpolar Health 84. Proceedings of the $6^{\text {th }}$ International Symposium on Circumpolar Health, Universiy of Washington Press, Seattle and London, pp. 261-265.
Iaconelli (S.), Hladik (C.M.), Pasquet (P.), Simmen (B.), 1998. Tannin perception: Comparative studies on taste thresholds in a non-human primate and among samples of human populations. 17th Congress of the International Primatological Society. Antananarivo, Madagascar (Abstract $\mathrm{n}^{\circ} 364$ ).

IACONELLI (S.), 1998 (en préparation). La perception des tannins et autres produits secondaires che les Primates.

KRUPNIK (I.), 1993. Arctic adaptation: Native Whalers and Reindeer Herders of Northern Eurasia. University Press of New England, Hanover and London.

Lecoutre (B.), Poitevineau (J.), 1996. LeBayésien, Windows software. Supplied in Lecoutre B. 1996, Traitement statistique des données expérimentales : des pratiques traditionnelles aux pratiques bayésiennes. C.I.S.I.A., Saint-Mandé, France.

Malet (C.), 1990. Les peuples du Nord aujourd'hui. Boréales, Revue du Centre de Recherches Inter-Nordiques, 40/45: 1-384.

MALET (C.), 1993. A l'interphase de la médecine et de l'anthropologie: propos sur la santé des peuples autochtones du Kamtchatka et de la Tchoukotka. Questions sibériennes, Sibérie III, pp. 207-222.

Malet (C.), 1995. Notes sur l'environnement de la Basse-Kolyma avec un inventaire des mammifères, des oiseaux et des baies qu'on peut y observer. Boréales, Revue du Centre de Recherches Inter-Nordiques, 62/65: 159-172.

MaLet (C.), 1998. Réflexions sur la santé des peuples de Yakoutie: approche anthropologique et médicale. Boréales, Revue du Centre de Recherches Inter-Nordiques. 74/77 : 67-110.

Robbe (B.), HLadiK (C.M.), 1988. Perception et consommation du sel dans la société Inuit de la côte orientale du Groenland. Journal d'Agriculture Traditionelle et de Botanique Appliquée, 35 $67-75$.

Roвве (P.), 1994. Les Inuit d'Ammassalik, Chasseurs de l'Arctique. Mémoires du Muséum National d'Histoire Naturelle, 159: 1-389.

Robert-Lamblin (J.), 1986. Ammassalik, East Greenland - end or persistance of an isolate ? Anthropological and demographical study on change. Meddelelser om Grönland, Man and Society, $10,168 \mathrm{p}$

Robert-Lamblin (J.), 1998. Alimentation et pratiques médicales traditionnelles des populations de la Basse-Kolyma (Yakoutie). Boréales, Revue du Centre de Recherches Inter-Nordiques, 74/77: 31-65.

SAS Institute, 1994, SAS/STAT user's guide, version 6, 4th edn, Vol 2., SAS Institute Inc, Cary. 
Simmen (B ). HLADIK (C.M.),1993. Perception gustative et adaptation à l'environnement nutritionnel des Primates non humains et des population humaines. Bulletins et Mémoires de la Société d'Anthropologie de Paris, n. s., 5: 343-354.

Wrangel (F. DE), 1843. Le Nord de la Sibérie. Voyage parmi les peuplades de la Russie Asiatique et dans la Mer Glaciale. Librairie d'Amyot, Paris, 2 tomes., 382 et 393 p

Accepté le 22/12/1998 